\title{
A LARGE MOTION SUSPENSION SYSTEM FOR SIMULATION OF ORBITAL DEPLOYMENT
}

T. M. Straube" and L. D. Peterson ${ }^{\dagger}$

\author{
Center for Aerospace Structures \\ and Department of Aerospace Engineering Sciences \\ University of Colorado at Boulder \\ Boulder, CO 80309-0429
}
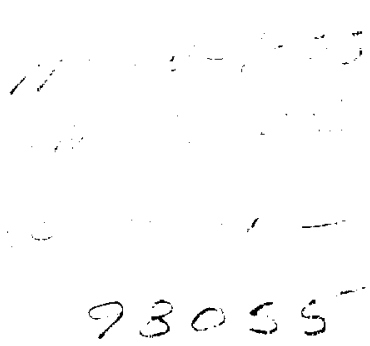

\begin{abstract}
This paper describes the design and implementation of a vertical degree of freedom suspension system which provides a constant force off-load condition to counter gravity over large displacements. By accommodating motions up to one meter for structures weighing up to 100 pounds, the system is useful for experiments which simulate the on-orbit deployment of spacecraft components. A unique aspect of this system is the combination of a large stroke passive off-load device augmented by electromotive torque actuated force feedback. The active force feedback has the effect of reducing breakaway friction by an order of magnitude over the passive system alone. The paper describes the development of the suspension hardware and the feedback control algorithm. Experiments were performed to verify the suspensions system's ability to provide a gravity offload as well as its effect on the modal characteristics of a test article.
\end{abstract}

\section{INTRODUCTION}

Orbital missions are always preceded by ground testing to validate the mechanics of spacecraft structures. A variety of devices and apparatus are used to model some aspect of the orbital environment so as to improve the accuracy of the ground test. These simulations often model orbital temperature, radiation, vacuum level, and gravity. This research focuses on a method for simulating a structure's response to an orbital gravity environment. Experimentally, this is accomplished by producing a constant force gravity off-load using a mechanical suspension system in a $1-\mathrm{g}$ laboratory.

To simulate the unrestrained motion that occurs on orbit. a suspension system must exactly support the weight of the structure throughout the entire region of motion while introducing no

\footnotetext{
- Graduate Research Assistant. Student Member AIAA

'Assistant Professor, Senior Member AIAA, Associate Member ASME

Copyright O1994 by T. M. Straube and L. D. Peterson. Published by the American Institute of Aeronautics and Astronautics, Inc. with permission.
}

extraneous constraint forces on the test article. True free-free boundary conditions are not achieved however, because gravity loading creates sagging and stresses due to the weight of the structure. This loading can be minimized by support the structure at a number locations. This paper describes a single point off-load system which can be used singularly or in conjunction with numerous systems to allow terrestrial simulations of spacecraft docking, deployment, or assembly [1]. The system allows for motions up to 1 meter for test articles weighing up to 100 pounds. This paper describes the implementation and verification of the first generation hardware, support equipment, and controller of the suspension system. The design utilizes a passive mechanism to statically off-load the test article weight and an electromotive actuated force control feedback loop to offset the passive inertia and friction.

Current off-loading techniques range from the very simple to the complex. The ensuing examination of these techniques will describe the more simplistic techniques in brief and then delineate the design of two passive and one active suspension system.

Simple techniques include very long cables, air bearings, and counterweights. Long cables have low pendulum stiffness which provides near zero constraining forces in a horizontal plane. The use of a static cable however eliminates vertical motion except in the case of very soft cords. This type of support is adequate for small ranges of motion (one to (wo inches) on high frequency structures ( $10 \mathrm{~Hz}$ or greater). Beyond this, the length of cable needed to reduce stiffness is prohibitive for most facilities [2]. The overhead clearance requirement can be reduced by changing from a linear spring element to a rotational spring which requires much less operating room [3]. Airbearing surfaces and tracks provide another method of producing simulated free-free motion in a horizontal plane. This method can be used in conjunction with a vertical degree of freedom system to remove constraints in three dimensions. Spherical air bearings also exist which provide rotational degrees of freedom through limited, approximately $20^{\circ}$, angular displacements [2]. Counterweights represent probably the simplest method of producing a vertical degree of freedom. The disadvantage here is the dou- 
bling of the system mass which must be accounted for when evaluating any results.

More advanced passive techniques include Zero Spring Rate Mechanisms (ZSRM) which produce a nearly constant force over a limited stroke. This zero stiffness can be produced in a number of ways. Mechanical concepts use springs configured to produce a negative stiffness element on a suspended test article. This negative stiffness is countered by a positive stiffness element to produce a region of near zero stiffness. One such device $[4,5]$ is able to support a $300 \mathrm{lbf}$ mass through a one inch deflection. The mechanism exhibited suspension modes at $0.16,5$, and $7 \mathrm{~Hz}$ with a breakaway friction level of 2.3 grams. Another type of passive system [6] utilizes an air lubricated pneumatic cylinder to support test structures of up to $350 \mathrm{lbm}$. The essentially zero stiffness of the supporting air column allows the test article to maintain a static deflection with zero return force. No suspension frequencies were exhibited between 0.2 to $10 \mathrm{~Hz}$. A linear electromechanical actuator is used to provide small centering stiffness at $0.1 \mathrm{~Hz}$ to keep the test article centered. The reported stroke of the device is 2.4 inches with a breakaway friction level of I gram.

Methods also exist which utilize active compensation in offloading a test structure. These include a system which utilizes a torsional spring to support a test article around an equilibrium position [3]. A motor using position feedback provides negative stiffness to compensate for the springs changing torque as the test article deflects. The control system was designed with a friction compensator. The concept can be extended to three dimensions using three suspension devices positioned at the vertices of an inverted tripod and connect to the test article via three cables which attach to a single point. Tests off-loaded a $52 \mathrm{lbm}$ tip mass on a 41 inch cantilever beam through an 18 inch range of motion. The motor was found to be effective in reducing suspension system damping. Problems included electronic noise associated with the motor controllers and friction in the system.

From the above mentioned experiences and results published in other literature, $[2,5]$ a number of guidelines were developed for this research. These goals were designed to ensure an accurate representation of the structures on-orbit mechanics.

Consider first the suspension system mass which affects the rigid body modes of the test article. A target for the suspension system equivalent mass was established as $5-10 \%$ of the test article mass. It is also important that the vibration frequencies of the suspension system be well separated from the fundamental modes of the test structure. Failure to achieve this requirement may cause modal coupling between the structure and the suspension system, which can cause a frequency shift or change in the mode shapes of the structure. Prior work indicates that suspension modes should be no greater than one-fifth of the supported structures fundamental mode. System friction can also limit the fidelity of a simulation by inhibiting performance in two ways; increased damping in modes of the suspended structure and a reduction in rigid body acceleration.
The targeted system load capacity was established as 10 to $100 \mathrm{lbm}$ to support various structures of interest. The baseline structure for the design was chosen as an eight bay Warren truss weighing $38.9 \mathrm{lbm}$ with a fundamental frequency near $50 \mathrm{~Hz}$. The defining requirements suggest a fundamental suspension frequency less than $10 \mathrm{~Hz}$ and system inertial mass on the order of 1 to $5 \mathrm{lbm}$.

A review of the literature and the system requirements suggested that current ZSRM technology would not provide the range of displacement desired in this research. A paper by L.F. Yang [7] however describes a ZSRM which could provide zero stiffness over several meters. This concept was selected as the passive mechanism for the current system. Results from active feedback techniques [3| show opportunity for improved performance over solely passive systems. Instead of using position feedback, this research has developed a system with dircct feedback of the off-loading force to sense disturbances at the test article. This combination of a passive system with active force feedback controller combines the advantages of both systems. In this arrangement, the passive system supports the static weight of the test article thereby relieving the active system load requirements. The active system provides precise user specified performance control through feedback and is capable of compensating for passive system inertia, stiffness, and friction. Additionally, the passive system, which is inherently stable, provides a safely mechanism in the event of active system failure.

The ZSRM consists of a torsional spring and a non-circular disk which link to the test article via a cable which wraps around the disk. This passive off-load acts in parallel to the active force delivered by a DC servo motor whose shaft is linked to the disk. A load cell in the support cable provides force fecdback to a Proportional Integral Derivative (PID) controller. The system design is shown in Figure 1.

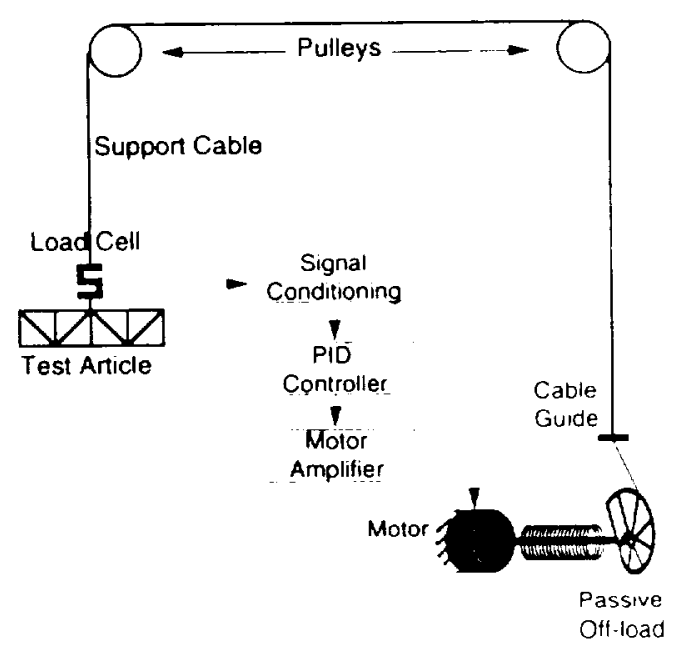

Figure 1: Suspension system schematic 
The following section describes the implementation of the passive-plus-active suspension system. This includes details of the passive off-load mechanism and the feedback control loop. The third section describes tests performed to optimize the feedback control scheme and evaluate rigid body acceleration characteristics of the suspension system. The fourth section examines the suspension system's effect on the modal characteristics of a suspended structure. The last section discusses final results and possible improvements for future development.

\section{SYSTEM IMPLEMENTATION}

The suspension system consists of two distinct subsystems: the passive off-load device and the force feedback loop with electromotive actuation. The design methodology and rationale of each are described in the following sections.

\section{Passive Sub-System Design}

The passive subsystem, a ZSRM capable of large ranges of motion, consists of a torsion spring and a non-circular disk [Figure 2].

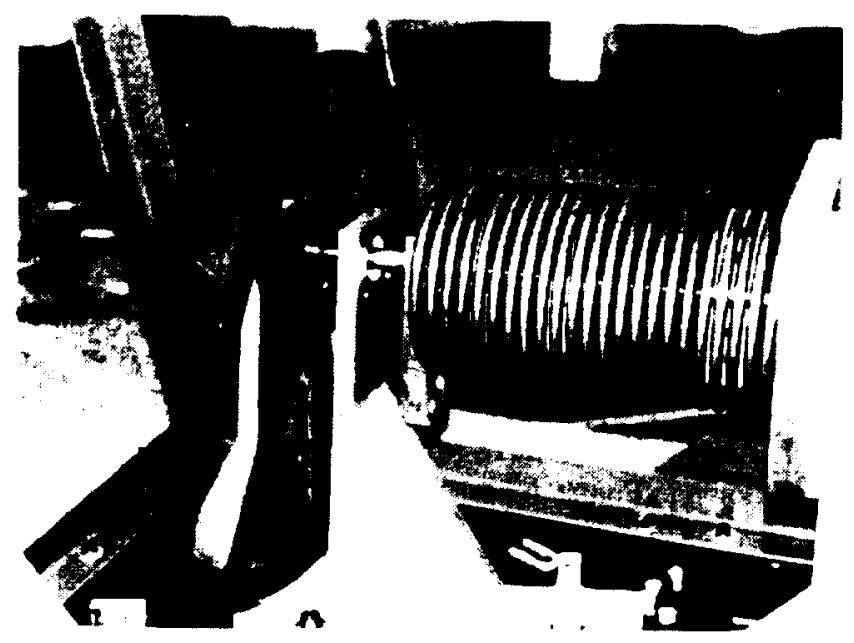

Figure 2: Photo of the large stroke ZSRM

Given an initial rotation, $\beta_{0}$, the torsional spring produces a torque, $M_{\text {pring }}$, on the disk given by,

$$
M_{\text {spring }}=\left(\beta_{0}+\theta\right) \kappa
$$

where $\theta$ is disk rotation and $K$, is the spring rate. This is offset by the equal magnitude moment created by the cable force around the center of the disk.

$$
M_{\text {tah/c }}=\Psi \cdot D(\theta)
$$

$D(\theta)$ is the perpendicular distance from the cable to the center of the disk and $W$ is the weight of the test article. It should be noted that this distance is not the same as the radius of the disk,
$R(\theta)$, the point that the cable contacts the disk. To maintain static equilibrium, it is necessary that the two moments balance [Figure 3]. This establishes a relationship between the rotation angle of the disk, $\theta$, and the required moment arm of the disk at that rotation, $D(\theta)$ :

$$
\left(\beta_{0}+\theta\right) K_{1}=W \cdot D(\theta)
$$

As the disk rotates, the effective moment arm. $D(\theta)$, of the cable force changes such that the torque from the torsion spring is balanced.

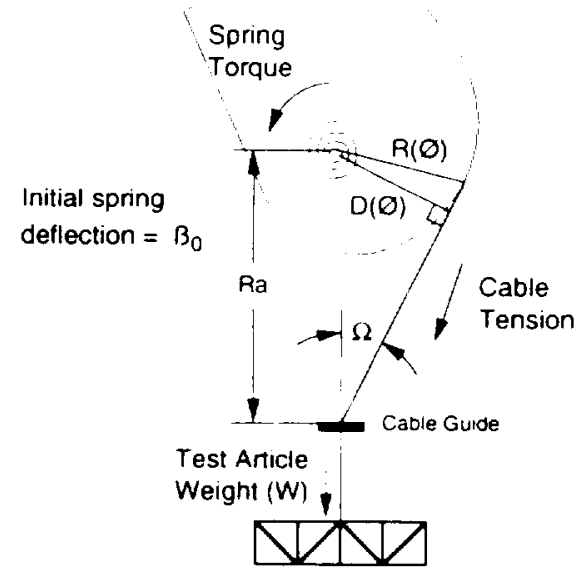

Figure 3: Spring torque balanced by the test article weight

The equations for this type of zero spring rate mechanism were developed in a paper by L.F. Yang [7]. The concept is also referred to in a book on classical mechanisms by N.P. Chironis [8]. Yang's equations for the disk profile were used to determine the coordinates of the disk shape. From the equations which define the disk profile, five independent parameters completely specify the entire passive system configuration given a specific test structure weight, $W: K_{s}$ is the spring rate of the torsion spring, $\beta_{0}$ is the initial deflection of the spring, $\theta_{\mathrm{up}}$ and $\theta_{\text {down }}$ are the furthest rotation of the disk from the nominal position, and $R_{a}$ is the distance from the center of the disk to a stationary cable guide. Once created, the system can support a test article of any weight provided a new torsion spring is used to maintain the parameter, $\alpha=W / K_{s}$. Current configurations were designed for $W=$ $38.9 \mathrm{lbm}$, the weight of the baseline test article.

The design criterion for the disk were primarily based on realizing a one meter stroke, the deflection limitations of the torsional spring. and the torque characteristics of the motor. was achieving which allows one meter of motion. The stroke of the system is simply the length of the useful curvature of the disk profile, which must maintain a convex curvature through the region of cable contact. Thus a disk with larger average radius needs less angular deflection to achieve a given stroke than a disk with a small radius. The disk used throughout these experiments utilizes a spiral curvature which deflects through more 
than $360^{\circ}$. Although this added complexity to the design and machining, it allowed for a smaller average disk radius. Because the motor and torsional spring torque is delivered through the disk, a smaller disk allows a smaller, and less expensive motor and spring to produce the same command authority over the test article as larger components. This size is limited by the rotational deflection of the spring which can reach very high stress level and experience nonlinear effects when rotated large angles. As a result, a maximum rotation was established as $540^{\circ}$. To reduce the number of spring coils and average coil diameter, the spring rate, $\mathrm{K}_{s}$, was limited to $5.0 \mathrm{Nm} / \mathrm{rad}$.

Another consideration was the friction between the cable and the cable guide. The cable produces a side load, $\mathrm{F}_{s l}$, on the cable guide equal to:

$$
F_{s}=2 W \sin \left(\frac{\Omega}{2}\right)
$$

where $\Omega$, is the cable incidence angle [Figure 3]. Because the friction force is proportional to the loading force, the angle $\Omega$ needs to be kept as small as is practical. The angle can be reduced by moving the cable guide further from the disk center, but this forces the system housing to be larger in order to support the cable guide. The disk size can also be reduced to lessen the angle so long as the other requirements are satisfied. A number of designs which met the guidelines were considered. Table I summarizes the parameter for the disk which is precision machined from a single $3 / 8$ " aluminum tooling plate.

\begin{tabular}{|l|l|}
\hline Inital spring deflection & $\beta_{0}=360^{\circ}$ \\
\hline Maximum angular rotation up & $\theta_{u p}=290^{\circ}$ \\
\hline Test artıcle translation up & $0.52 \mathrm{~m}$ \\
\hline Maximum angular rotation down & $\theta_{\text {down }}=160^{\circ}$ \\
\hline Test article translation down & $0.56 \mathrm{~m}$ \\
\hline Distance from disk center to cable guide & $\mathrm{Ra}=0.165 \mathrm{~m}$ \\
\hline Frequency parameter & $\alpha=1.907 \mathrm{rad} / \mathrm{m}$ \\
\hline Maximum disk radius & $0.2394 \mathrm{~m}$ \\
\hline Minimum disk radius & $0.04227 \mathrm{~m}$ \\
\hline Cable guide incident angle & $\Omega=27.9^{\circ}$ \\
\hline Final design weight & 1.6 pounds \\
\hline
\end{tabular}

Table 1: Disk design parameters

The next step was the selection of the torsional spring. The disk shape dictated a spring rate of $K_{s}=4.5 \mathrm{Nm} / \mathrm{rad}$, for the 38.9 Ibm test article. The spring's maximum rotation is given by,

$$
\theta_{\text {max }}=\beta_{0}=520^{\circ}
$$

which determines the springs maximum restoring torque:

$$
T_{\max }=\theta_{\max } K_{s}=40.84 \mathrm{Nm}
$$

From these parameters, the spring, made from oil tempered wire, [Figure 4], was designed following a standard industry methodology [9].

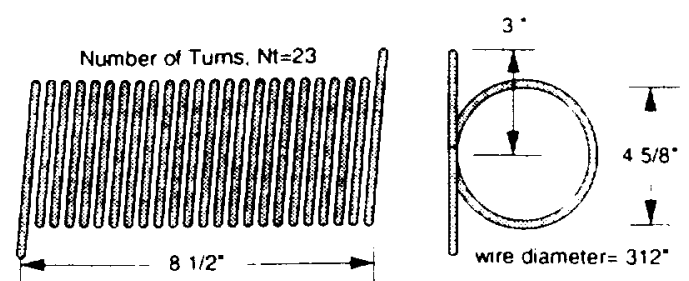

Figure 4: Torsional spring design drawing

An additional issue in the spring design was inter-coil friction during extreme deflection. As the spring deflects, the coils move closer together. Eventually they touch, creating friction. The design of the spring pitch allows for coil compression that takes place during rotation so that a gap remains between the coils even upon furthest deflection.

Testing conducted with the spring showed that it sagged under its own weight and deflected laterally as torque was applied. These factors made it necessary to support the spring with a mandrel. Initially, a single piece mandrel [Figure 5] was placed inside the spring for support. As the spring deflects, it binds in certain spots along the mandrel. Because the mandrel is free to rotate upon its support shaft, this in itself does not cause a problem. The difficulty arises because one end of the spring remains fixed as the other rotates. If the spring binds on the mandrel in multiple locations which are moving at various rates, large amounts of friction are generated. Two steps were taken to alleviate this problem. First, a new mandrel consisting of four one inch pieces [Figure 5] replaced the full length mandrel. This allowed the mandrel pieces to rotate with the portion of the spring that it supported, minimizing any rotational differences. Second. the entire spring and mandrel pieces were coated with a friction reducing treatment [10]. A noticeable but not complete elimination of friction was achieved.

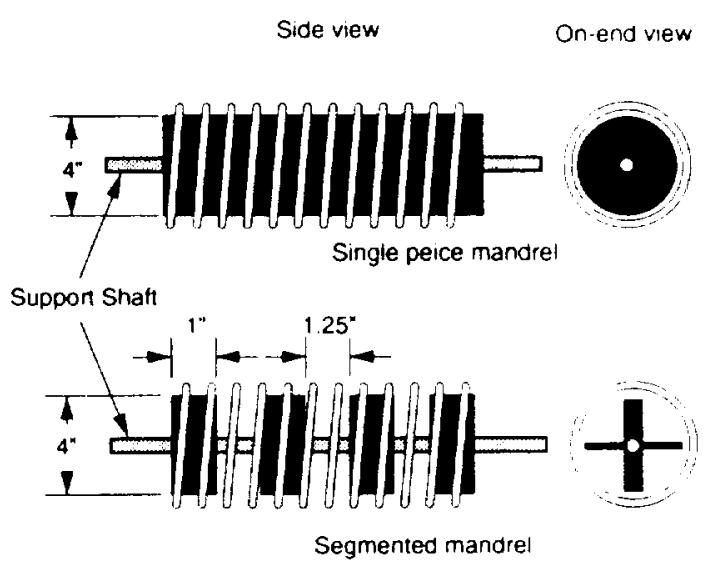

Figure 5: Solid and piecewise mandrel designs

The disk is mounted to a shaft which is directly linked to the drive shaft of the motor. The same shaft supports the spring mandrel. All of this hardware, as well as the wire guide, is supported by a housing [Figure 6] which is hard mounted to the 
floor. A 1/16" aircraft cable wraps around disk and passes over a small low friction bearing which act as a cable guide. The cable then passes through two overhead pulleys affixed to the ceiling and down to the fixture point on the test structure.

The passive system has the effect of adding the equivalent of approximately $3 \mathrm{lbm}$ to the test article from all components including the load cell, aircraft cable. disk inertia, spring inertia. Breakaway friction for the system is highly dependent on the deflection of the spring indicating that spring binding is still a problem. At some rotations the friction is as high as $1 \mathrm{lbm}$.

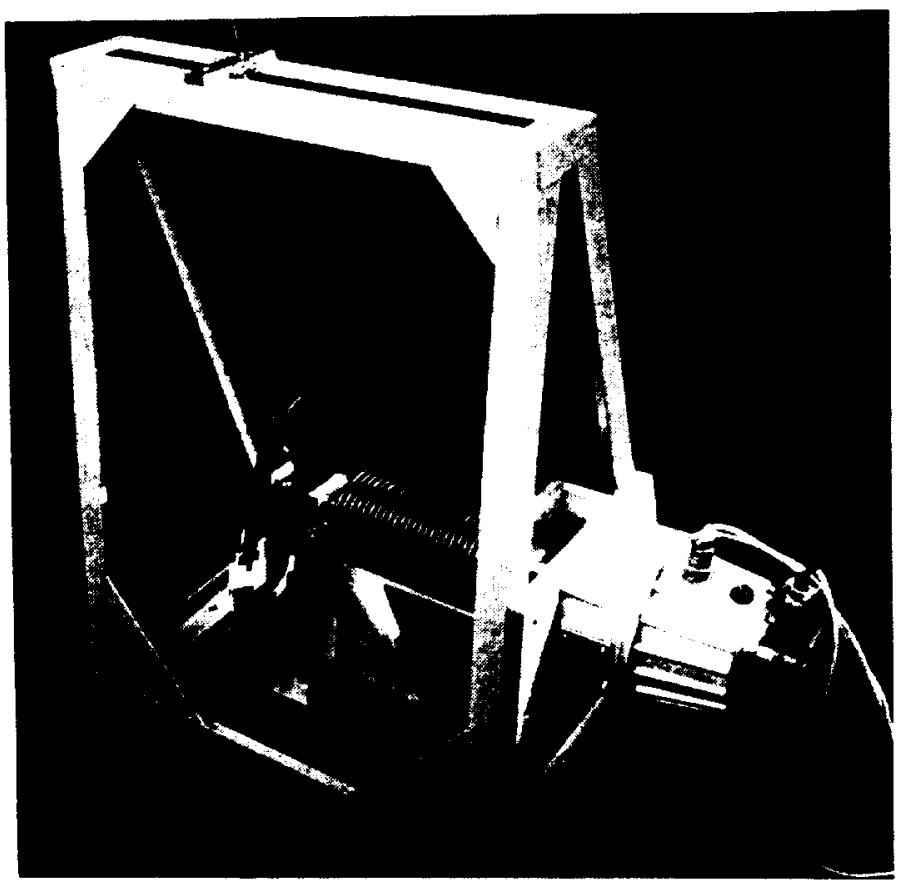

Figure 6: Photo of the passive off-load system in its housing

\section{Active System}

The active system [Figure 7] is driven by force feedback from a load cell in the cable path near the test article. Accurate gravity off-loading requires that the cable tension equal the weight of the structure. Any input force on the test article will cause the cable tension to deviate from this value and create an error signal. The controller reacts to the error signal and commands the servo motor to respond such that the acceleration of the test article returns the cable tension to the test article weight.

Control actuation is achieved via a brushed DC servo motor and switching servo amplifier. The characteristics for the motor and amplifier chosen are shown in Table 2.

\begin{tabular}{|c|c|}
\hline \multicolumn{2}{|c|}{ Motor Characteristics: } \\
\hline Manufacturer & PMI Motion Technologies \\
\hline Motor type & IRI $6 \mathrm{M} 4 \mathrm{CH}-1$ \\
\hline Peak torque & $\$ 374 \mathrm{oz} \cdot \mathrm{in}$ \\
\hline Stall torque & $51002 \cdot \cdot$ in \\
\hline Continuous stall current & 764 amps \\
\hline Clogging torque & $00 z . \cdot 1 n$ \\
\hline Friction torque & $11002 \cdot$ in \\
\hline Moment of inertia & $0.075 \mathrm{oz} \cdot$ in sec $\bullet$ sec \\
\hline \multicolumn{2}{|c|}{ Amplifier Characteristics: } \\
\hline Manufacturer & Advanced Motion Solutions \\
\hline Type & $30 \mathrm{~A} 20 \mathrm{AC}$ \\
\hline Peak current & 30 amps \\
\hline Max. continuous current & $15 \mathrm{amps}$ \\
\hline Bandwidth & $2.5 \mathrm{kHz}$ \\
\hline
\end{tabular}

Table 2: Motor and Amplifier Characteristics

A digital controller [11] takes the current load cell signal and compares it against a reading taken during initialization to form the controller error signal. The control loop [Figure 7] executes at $150 \mathrm{~Hz}$ until the user manually terminates the controller or the test article translates beyond preset bounds established by the user. In the first step of the control loop the most recent data from the load cell is read into memory from the 16 bit analog to digital converter. This voltage is converted to a force and subtracted from the initial load cell reading to form the controller error signal. A signal dead band zeros any signal whose absolute value is below a user defined level. Any error greater than the dead band level is not effected.

The error, now in pounds, enters the Proportional, Integral, Derivative (PID) logic block. Its voltage output is based on a proportional, integral, or derivative relationship with the load cell error. The exact formulation follows:

$$
Y_{\text {out }}=K_{p} X(n)+K_{d} \frac{X(n)-K(n-1)}{\Delta T}+K \sum_{k=1}^{n} \frac{X(k)+X k-1)}{2} \Delta T
$$

where $X(n)$, is the load cell error at each discrete time step, $\Delta T$, after processing by the dead band. $K_{p}, K_{d}$, and $K_{I}$, are the proportional, derivative and integral gains. The voltage from the PID passes through a limiter, which limits the output voltage for safety reasons, before being processed by the disk radius compensation.

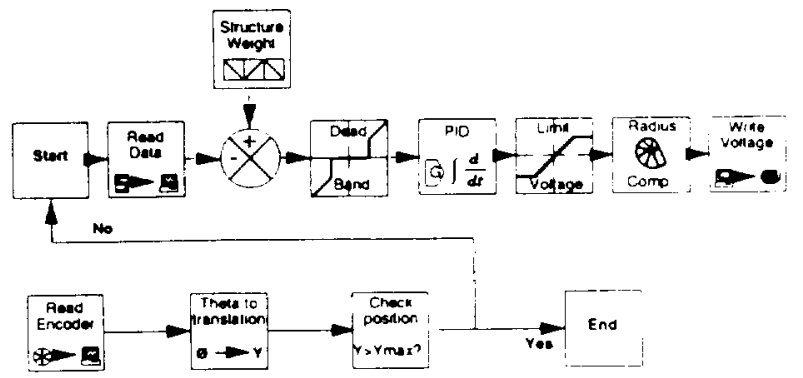

Figure 7: Control logic diagram 
Because the disk's radius is not constant, a given motor torque will produce varying forces on the test article, depending on the disk's angle of rotation. This condition causes the controller to behave inconsistently as the test article translates. The radius compensation scales the torque produced by the motor so that the resulting force on the test article is independent of disk orientation. Finally, the control voltage is sent to the 12 bit digital to analog converter and out to the amplifier.

Control feedback is provided by a load cell which links the cable and the test article. This load cell must be able to quickly respond to any changes in cable tension and also endure the wide range of loads from different test articles. A load cell was chosen with a $100 \mathrm{lbf}$ range with and output of 0.05 volts/lbf. An in-line bi-polar amplifier provides the load cell power and also amplifies the output signal. After zeroing the amplifier prior to each test, the final transducer sensitivity is 4.9975 volts out of the amplifier per $100 \mathrm{lbf}$. tension applied to the load cell. Before being digitized, the signal passes through an anti-aliasing low pass filter and gain stage before digitization. The signal conditioner uses a low-pass eight pole Butterworth filter which was set to a $30 \mathrm{~Hz}$ cutoff frequency as determined by the $150 \mathrm{~Hz}$ sampling rate and the resolution of the digitizer.

The other quantity monitored by the controller is test article position. From the known profile of the disk and approximate relation was established between the disk rotation angle and the translation of the test article. An encoder on the DC motor provides the rotation angle of the disk. Empirical results showed that this measurement was reliable to a resolution of $\pm 0.002 \mathrm{~m}$. The position was monitored by the controller and enacted shut down if the test article exceed preset limits on its motion.

\section{RIGID BODY EXPERIMENTATION}

A number of tests were performed to determine its effectiveness in off-loading gravity for rigid body elements. Initial tests determined the best controller configuration and then simulations were performed with various levels and types of input forces.

\section{Test Articles}

All of the rigid body tests were performed using a lumped mass test article. This ensured that any observed low frequency modes were from the suspension system. For the passive-plusactive system a $38.9 \mathrm{lbm}$ solid steel cylinder 5.5 inches in diameter and 5.75 inches tall was used. An eyebolt was tapped into one end to provide an attachment to a hook which extended from the bottom of the load cell.

\section{Inout Force Types}

Tests performed with the lumped mass utilized two types of input forces. The first was a "virtual" force which causes the controller to act as if a force has been applied to the test article. To achieve this a small perturbation is added to the load cell signal inside the controller. When this new signal is compared to the initial load cell reading of the test article weight an error signal is produced and sent to the PID controller. In this way any input force can be synthesized.

This type of loading was used during controller configuration to avoid the random aspect of a physically applied load. The repeatability provided a very reliable method of comparing system response for varying controller settings.

Real input forces were used to compare the suspended test article's theoretical on-orbit and measured response to an input force. Constant force tests were performed by initializing the controller with the base test article weight and then, with the controller on, placing additional mass on the test article. The additional weight creates a downward force on the test article causing both test article and additional mass to accelerate.

As mentioned previously, this type of force input is subject to random errors introduced as transient impact forces and experimenter disturbances. Although the net input force of these inputs is small, they also have an effect through the moments they induce. Any off-center forces create a moment which tends to cause the test article to swing, exciting some of the suspension modes.

\section{Controller Tuning}

A number of tests were necessary to optimize the PID control gains and the load cell error dead band level to produce the best simulation of on-orbit dynamics. Specifically each type of gain. proportional, derivative, and integral were tested for system response characteristics.

The final scheme utilizes integration only feedback. It is the only feedback mechanism that is capable of compensating for the friction in the passive mechanism. Results with integration gain are presented first and with a brief discussion other gains following.

Figure 8 shows a typical response to a constant force with integration gain only. At time 0 , the constant input force is applied and creates an error in the load cell reading. Test article motion has not yet started because of friction in the passive system From 0 to 0.5 seconds the controller integrates the error and produces and increasing output voltage. At 0.50 seconds, the controller output commands the motor torque to a level which compensates for the friction. The resulting test article acceleration reduces the load cell error to a near zero average. Because the errors are small, the control output remains at a near constant level for the remainder of the test.

The resulting motion of the test article is also shown. From 0 to 0.5 seconds little motion occurs. At 0.5 seconds the active system has completely compensated for the passive friction and a constant force produces a nearly constant acceleration. The test article position was calculated from the motor encoder data. A low pass filter was used to remove any high frequency content after which the signal was differentiated to get velocity. An ap- 
proximate constant acceleration was derived from the slope off a least squares fit to the velocity data.

Although a nearly constant acceleration is produced, problems exist with this scheme. The most obvious is the delay associated with integration controller, which results because the controller cannot instantly overcome system friction when a load is applied. The time needed to overcome the friction and have the test article reach a constant acceleration will be termed the "integration lag".
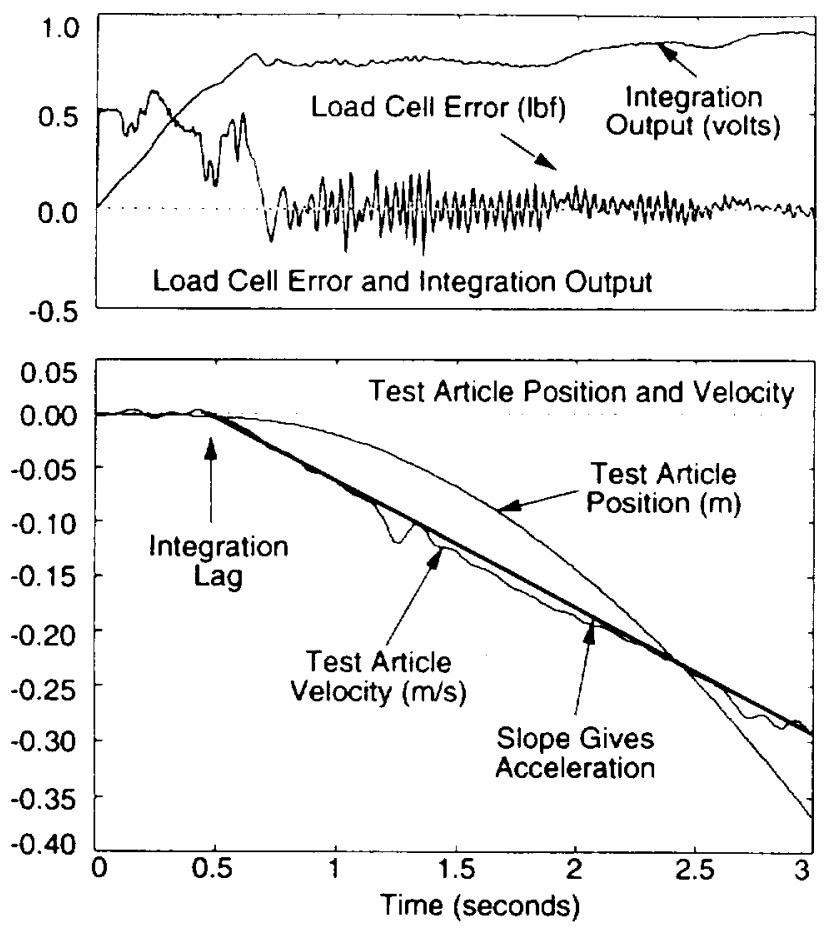

Figure 8: Typical rigid body response with integration gain

A number of steps can be taken to reduce this lag. Reducing friction in the passive mechanism will require less output torque resulting in a faster response. Another possibility is raising the integration gain which will increase the controller output at a faster rate. This solution can create a problem with the system's stability. Figure 8 shows that the load cell error does not go to zero but instead oscillates about zero with an unidentified $27 \mathrm{~Hz}$ suspension mode. This mode was seen in many of the experimental results. It can become critical if it interferes with the modes of a test structure. The mode also became unstable when an integration gain of 15 volts/lbf or greater was used. For gains of 10 volv/lbf the mode was well behaved and generally damped out.

Figure 9 shows the integration lag and the relative error between the measured and theoretical acceleration for two tests using different values of integration gains. In one case a virtual force of $-0.5 \mathrm{lbf}$ was applied to the $38.9 \mathrm{lbm}$ test article with dead band level set to $0.01 \mathrm{lbf}$. The other test used a real $-0.65 \mathrm{lbf}$ force applied to the same test article level.
Both tests indicate that with a gain above 2 volts/lbf the acceleration can be expected to be within $10 \%$ of the predicted value. Below a gain of 2 volts/lbf the response becomes very delayed and does not match the theoretical acceleration. The optimum range of gains is 4 to 10 volts/lbf.

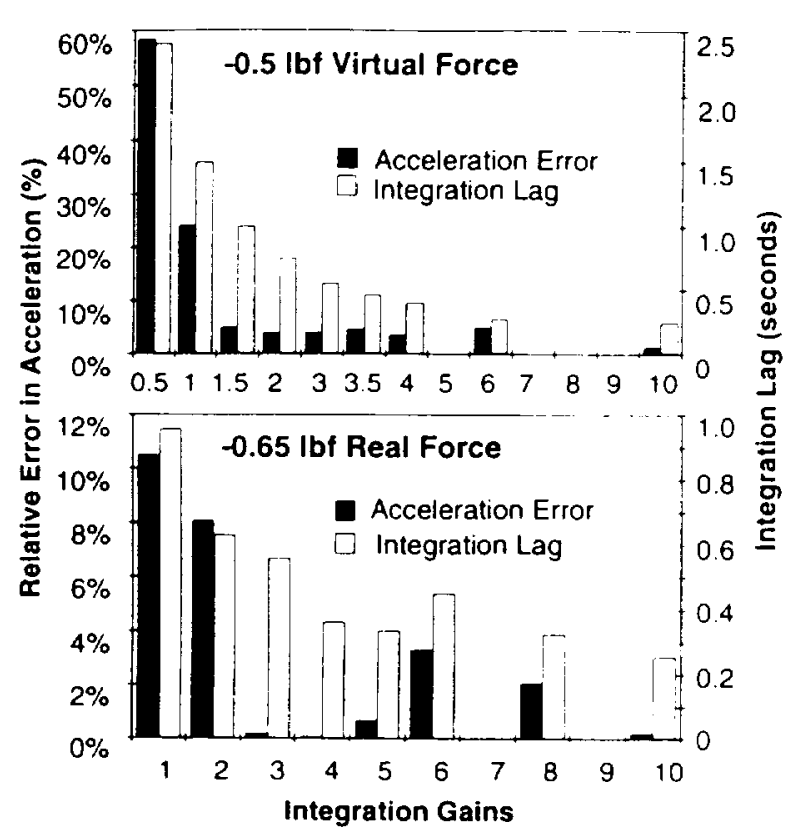

Figure 9: Acceleration error and time lag at various integration gains

Because of slight fluctuations in the load cell signal and noise during signal transmission there is a random component in the load cell signal. Although effects vary from test to test, the error commonly exhibits a bias, when integrated by the controller, can produce a command signal large enough to initiate motion in the test article

Because of friction inherent in the passive system, the motor must continuously produce a torque to offset the friction force. Only integration gain is able to provide this type of compensation. As an external force is applied to the test article, a load cell error appears. A proportional gain will respond by providing an output which sends the test article in motion. However, as the test article accelerated the load cell error drops to zero with the proportional controller also going to zero. With no lorque to offset friction the test article slows down again creating a load cell error, driving the system into oscillation.

A different problem occurs with the derivative gain. It can only respond to a change in the load cell reading. The derivative gain can not respond to a constant error, only changes in error. As a load is applied the system responds with an output which accelerates the test article. This relieves the error and gives a negative derivative which causes an output in the opposite direction which accelerates the test article in the opposite direction, again establishing an vibrating system. Both of these effects were observed in experimental tests. These tests showed that, by 
themselves, proportional and derivative gain cannot compensate for the system friction.

However, as indicated by the results of the above test results, integration control only may not be the optimum controller for the system. Utilizing proportional and derivative gain may help solve the problems by providing a more rapid response as a disturbance is initiated thereby reducing integration lag. Future work aimed at a full PID controller or perhaps some other controller scheme could reduce lag and provide a more stable response.

The dead band in the controller is used to reduce or eliminate the drift problem. Measurements of integration drift were taken with dead band set to $0.00,0.01$ and $0.02 \mathrm{lbf}$ [Figure 10]. Without a dead band all of the system noise passes to the integrator and integration drift is very significant. The $0.01 \mathrm{lbf}$ dead hand allows a small amount of noise but greatly reduces the output drift. With a dead band of $0.02 \mathrm{lbf}$, the noise is almost completely filtered out producing a very small integration drift. Although a large dead band produces a small drift, other factors must be considered. The system can not respond to any force smaller than the dead band level, thereby reducing the system sensitivity. The optimum dead band is the smallest level which reduces the integration level to an acceptable value for the experiment being conducted. For instance, drift will be more important in a slow deployment experiment during which the error will have a long period of time to grow and effect the test article motion.

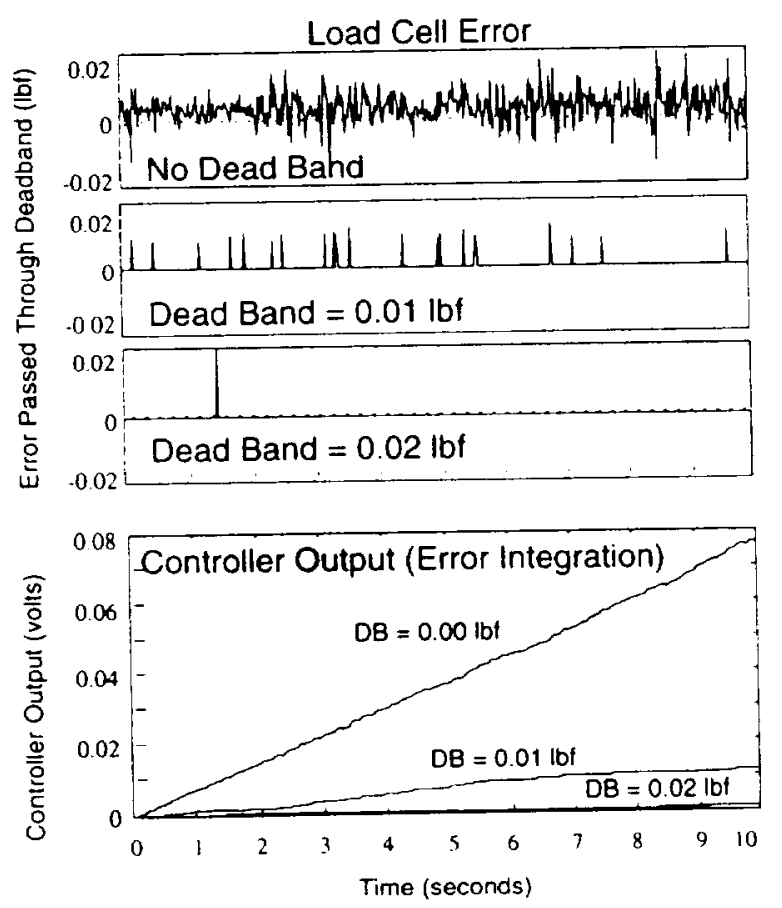

Figure 10: Effect of Dead Band on integration drift

\section{Force Response Test}

After determining how the system response changes with various control configurations, additional tests were performed to quantify the systems ability to track different levels and types of forces.

For these tests, the suspension system off-loaded the 38.9 lbm with a control dead band setting of $0.01 \mathrm{lbf}$. A constant real force was applied in varying magnitudes by placing different sized masses on the test article. The test range is controlled by the controller, which monitors test article position and shuts down after the preset displacement has been reached, which for these tests is $0.5 \mathrm{~m}$. Upon shut down the controller records total test time. Values of load cell error, controller output voltage, and test article position were recorded at each for each pass through the control loop.

At each force level, controller configurations with gains of 4 and 10 volts/lbf were used. Time lag and relative acceleration errors are shown for both gain tests in figure 11 .

Both gains produce acceleration accurate to within $10 \%$ down to force levels near $0.25 \mathrm{lbf}$. Between 0.1 to $0.25 \mathrm{lbf}$ the acceleration performance deteriorates and error and lag increase. Below 0.1 lbf no significant motion was record. The establishes a breakaway force level near $0.1 \mathrm{lbf}$. As comparison, with active feedback turned off, passive off-load only, the breakaway friction level is near $1.25 \mathrm{lbl}$ meaning an improvement in system sensitivity of an order of magnitude.

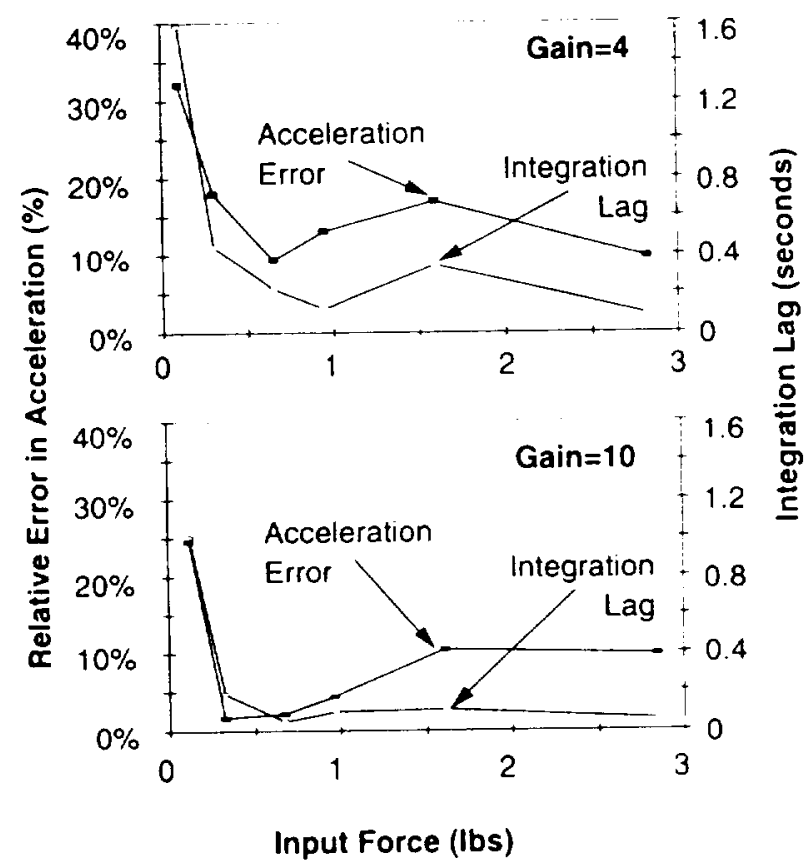

Figure 11: Test article response to various force levels 


\section{Modal Analysis of a Suspended Structure}

To determine the suspension system's effect on the vibrational characteristics of a suspended test structure, a modal survey was performed on a test article suspended by a static line and by the suspension system.

\section{Test Article Selection}

A $38.9 \mathrm{lbm}$ highly flexible test article [Figure 12] was designed to have a relatively low first bending frequency near 10 $\mathrm{Hz}$ in one transverse direction. The other direction has much higher stiffness to elevate the frequencies, above $150 \mathrm{~Hz}$, out of the bandwidth of the modal test. This eliminates any coupling between orthogonal modes and ensures that no out of plane modes appear in the frequency response function. The beam was also designed with a relatively short length to reduce sag in a one $\mathrm{g}$ field. A simple MSC/NASTRAN model was used to estimate the first and second bending modes which were predicted to be at $7.6 \mathrm{~Hz}$ and $71 \mathrm{~Hz}$.

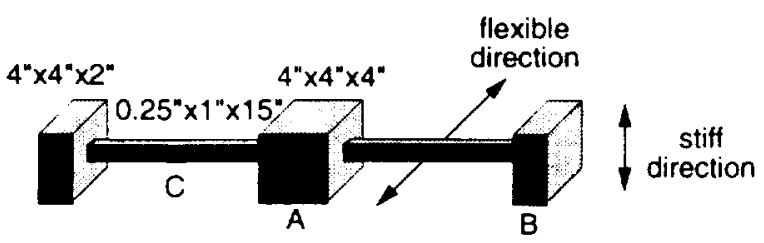

Figure 12: Highly flexible test article

\section{Modal Survey of Highly Flexible Test Articles}

Modal tests were performed with accelerometers at locations $A, B$, and $C$ along the beam aligned with the flexible direction of the beam. An impulse was delivered to the center mass using a modal hammer along the same axis as the accelerometers. Two tests were performed. For the first, the structure's flexible direction was aligned with the horizontal plane and the structure suspended from a single static cable at the center mass. These results [Figure 13] should represent the free-free modes of the structure because no external forces, including gravity, act in the horizontal direction. For the second test the flexible modes were oriented in the vertical direction with the structure suspended from the suspension system. The results are shown in the frequency response function [Figure 14], which gives he $\mathrm{dB}$ magnitude, phase, and coherence of the response

For the experiment in the horizontal direction, the first and second bending modes appear at $9.2 \mathrm{~Hz}$ and $77 \mathrm{~Hz}$. These compare closely to the predicted results from the finite element model, see table 3 . It should also be noted that three unpredicted peaks, near $41 \mathrm{~Hz}, 53 \mathrm{~Hz}$, and $98 \mathrm{~Hz}$ also exist. These are local modes in the beam which were not represented in the finite element model. Of more interest is an comparison of the modes from the actively suspended structure. It is apparent that the coherence and modal resolution are lost in the $30 \mathrm{~Hz}$ bandwidth of the suspension system controller. At these frequencies the modes appear highly damped. Above the bandwidth of the controller, the frequency response is relatively unaffected. No shift in frequency appears and the peak amplitudes are almost identical

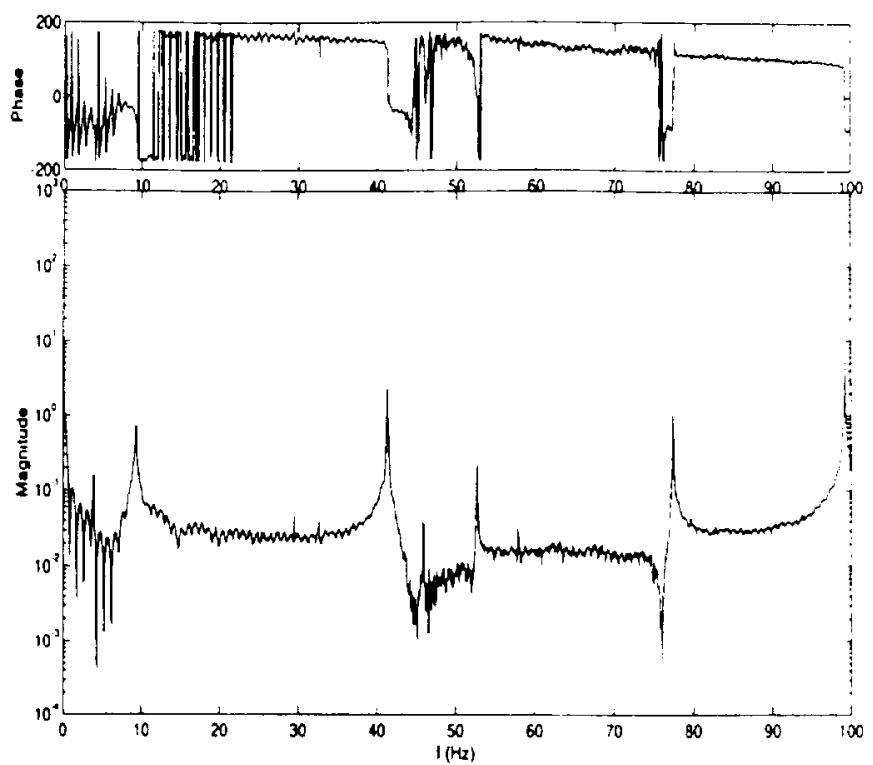

Figure 13: FRF of flexible test structure

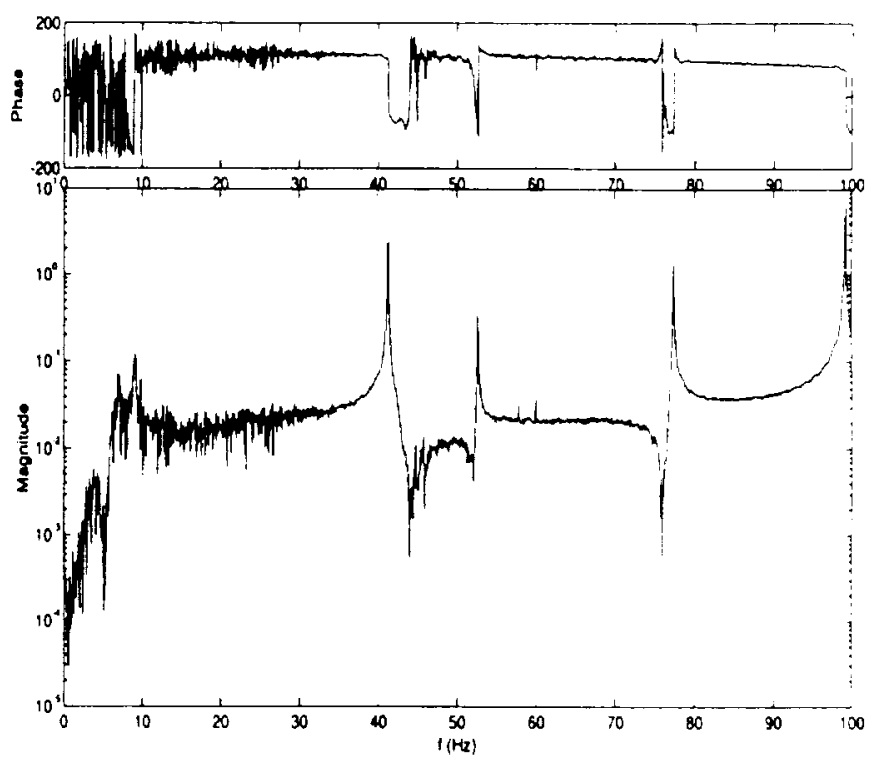

Figure 14: FRF of suspended flexible test structure

\begin{tabular}{|l|l|l|l|}
\hline & NASTRAN & Horizontal Test & $\begin{array}{l}\text { Actively Suspend- } \\
\text { ed Test }\end{array}$ \\
\hline First bending & $7.6 \mathrm{~Hz}$ & 9.2 & 9 (highly damped) \\
\hline Second bending & $71 \mathrm{~Hz}$ & 77 & 77 \\
\hline $\begin{array}{l}\text { Unidentified } \\
\text { Modes }\end{array}$ & no match & $41,53,98 \mathrm{~Hz}$ & $41,53,98 \mathrm{~Hz}$ \\
\hline
\end{tabular}

Table 3: Vibration frequencies of test article 


\section{DISCUSSION}

Summary of Current Capabilities

As presented, two major types of testing were performed with the one dimensional suspension system: rigid body acceleration and modal response of a suspended test article. While the passive-only system has a friction level of $1.25 \mathrm{lbf}$, active force feedback compensation is able to reduce this by an order of magnitude to $0.1 \mathrm{lbf}$. Simulations involving rigid body accelerations can be expected to be within $10 \%$ of the values achieved on orbit. The best performance was achieved using integral only feedback with a dead band of $0.01 \mathrm{lbf}$.

Integration lag was a problem identified with the integration only controller. Raising the control gain reduces the lag but also results in destabilization of the $27 \mathrm{~Hz}$ suspension mode. These iwo factors must be considered during controller configuration and result in some limitations of the suspension system. The lag can prohibit effective off-loading in experiments involving rapidly changing inputs. Nevertheless, the system can be useful in vertical deployment experiments and also vertical rigid body translation tests.

The results of the modal survey suggest that any attempt to examine test structure frequencies within the bandwidth of the controller will produce a highly damped response. For tests which involve structures with higher frequency modes, the results are within a few percent of the measured unrestrained values.

\section{Recommendations for Future Systems}

The three most critical problems identified in the currently implemented system are the integration lag, the passive subsystem friction, and the presence of the $27 \mathrm{~Hz}$ suspension mode. Refinements in the controller and the passive system could reduce or eliminate these problems. Suggestions for improvements in these areas follow.

Further testing of the system should be performed to better identify its vibrational characteristics. A frequency response function of the suspension system could be performed by inputting a sine dwell force into a lumped mass test structure suspended from the suspension system and measuring the resulting acceleration. This would identify suspension system modes and might help locate the source of the $27 \mathrm{~Hz}$ mode which was so pervasive during testing. Methods for reduce the effect of this mode could include eliminating it mechanically, if possible, or utilizing a stop band filter for the controller

The current controller frequency is software limited to 150 $\mathrm{Hz}$. With software upgrades the frequency can probably be raised to near $1000 \mathrm{~Hz}$. Modifications are feasible which can reduce the bandwidth to near $1000 \mathrm{~Hz}$. This would allow tests which examine changes in rigid body acceleration and test article modal response for various controller speeds and filter cutoff frequencies.
Further controller improvements include optimizing the PID gains. As mentioned, the optimum controller might include PID control. The primary goal of these refinements should be reducing the integration lag toward zero. Future tests should also directly measure the mass and stiffness that the suspension system adds to the test article.

Reducing the friction throughout the system would also improve system performance. Less friction would reduce the breakaway force needed and consequently reduce integration lag. A full study could be conducted to determine the various sources of friction and methods to reduce it. Friction sources include the overhead pulleys, the bearings used in the cable guide, the bearings in the disk support shaft, and the servo motor and encoder.

The greatest amount of friction is due to the torsional spring. The friction increases with spring deflection, when the coils contact each other and when binding on the mandrels becomes significant. To verify this, tests of the systems breakaway friction should be performed at positions with large and small spring deflections. Different mandrel designs may be able to reduce the contact friction. A spring utilizing more coil spacing or even a design with helical spiral coils might prevent adjacent coils from coming in contact with each other.

\section{CONCLUSION}

This paper presented a suspension system capable of offloading test structures for use in space construction type experiments. The research has verifies the implementation of the noncircular disk ZSRM to a large stroke off-load system. The passive system is capable of off-loading up to $100 \mathrm{lbf}$ over a range of $1 \mathrm{~m}$. The breakaway friction level is $1.25 \mathrm{lbf}$. With the addition of a force feedback integration loop controller this friction level was reduced an order of magnitude to $0.1 \mathrm{lbf}$. The response to constant force levels down to $0.25 \mathrm{lbf}$ is accurate to within $10 \%$ with the exception of the presence of the integration lag and a stability issue of the $27 \mathrm{~Hz}$ suspension mode. These problems, as well as the passive system friction, should be addressed in future refinements.

\section{ACKNOWLEDGMENTS}

This work was supported by NASA Headquarters Grant NAGW-1388 through the Center for Space Construction at the University of Colorado, and by NASA Langley research center grant NAG-1-1490 through the Center for Aerospace Structures. Special recognition should go to undergraduates $\mathrm{Mr}$. Robert "Kipp" Shearman and Mr. Andy Wobido who assisted in the research and experimentation. And finally. I would like to acknowledge the control system development efforts of Mr. Steve Kranock and Mr. David Smart.

\section{REFERENCES}

1. Straube, T.M.,"A Large Motion Suspension System for Experimental Simulation of Orbital Construction and Deployment, 
Masters Thesis, University of Colorado, Boulder, Colorado, 1993.

2. Hasselman, T. K., Editor, "AIAA/AFOSR Workshop on Microgravity Simulation in Ground Validation Testing of Large Space Structures, Final Report," EMA-TR-90-1153.

3. Quartararo, R., Dr. Hasselman, T.K., "Microgravity Suspension of Flexible Space Structures," Phillips Laboratory Final Report, November, 1991. PL-TR--91-3071.

4. Woodard, S. E. and Housner, J. M., "Nonlinear Behavior of a Passive Zero-Spring-Rate Suspension System." J. Guidance Vol. 14, No. 1. Jan.-Feb. 1991, pp. 88-89.

5. Cooley, V.M. and Giunta, A. A., "Laboratory Evaluation of Two Advanced Suspension Devices for Ground Vibration Testing of Large Space Structures," Proceedings of the 33th AIAA/ASME ASCE/AHS/ASC Structures, Structural Dynamics and Materials Conference, 1992, AIAA-92-2334-CP

6. Kienholz, D.A., “A Pneumatic/Electric Suspension System for Simulating On-Orbit Conditions," Presented at the Winter Annual Meeting of the American Society of Mechanical Engineers, Dallas, Nov. 25-30, 1990, 90-WA/Aero-8.

7. Yang L. F., Chew M. and Juang J. N., "Ground-Based Testing of the Dynamics of Flexible Space Structures Using Band Mechanisms," Doctoral dissertation of first author, Old Dominion University, Norfolk.

8. Nicholas P. C., Mechanisms and Mechanical Devices Sourcebook. New York: McGraw-Hill, Inc., 1991.

9. Spring Manufacturers Institute, Inc., Newcomb Spring Corp., Spring Design Manual," 1991.

10. Xylon, Nordic Ware, Commercial Coatings Division. Minneapolis, MN.

11. LabVIEW 2 Users Manual, National Instruments Corporation, September 1991 Edition, Austin, TX

12. Honeybee Robotics, "A Gravity Compensation System for Simulation of On-Orbit Telerobotic Operations," Final Report, New York, June 28, 1991, SBIR 90-1. 